This is the final peer-reviewed accepted manuscript of:

D. Fabbri, E. Berthet-Bondet, D. Masotti, A. Costanzo, D. Dardari and A. Romani, "Long Range Battery-Less UHF-RFID Platform for Sensor Applications," 2019 IEEE International Conference on RFID Technology and Applications (RFID-TA), Pisa, Italy, 2019, pp. 80-85, doi: 10.1109/RFID-TA.2019.8892187. The final published version is available online at DOI:

http://dx.doi.org/10.1109/RFID-TA.2019.8892187

Rights / License:

The terms and conditions for the reuse of this version of the manuscript are specified in the publishing policy. For all terms of use and more information see the publisher's website.

This item was downloaded from IRIS Università di Bologna (https://cris.unibo.it/)

When citing, please refer to the published version. 


\title{
Long Range Battery-Less UHF-RFID Platform for Sensor Applications
}

\author{
Davide Fabbri, Eliot Berthet-Bondet*, Diego Masotti, Alessandra Costanzo, Davide Dardari, Aldo Romani \\ DEI-ARCES, Alma Mater Studiorum Università di Bologna, Cesena, Italy - \{davide.fabbri21\}@unibo.it \\ *PHELMA, Institut Polytechnique de Grenoble, Grenoble, France
}

\begin{abstract}
This paper presents the architecture and design trade-off of a compact, programmable, battery-less UHF-RFID sensor tag for environmental monitoring. The node is based on a single UHF monopole matched to a dual-path rectifier for both energy harvesting purposes and wake-up radio capabilities. The based-band circuit includes a power management unit (PMU) to supply the microcontroller unit which is in charge of implementing the EPC Gen 2 Class 1 standard protocol fully working with European ETSI compliant readers. The prototype was built with off-the-shelf components in a $95 \times 31 \mathrm{~mm}^{2}$ PCB with low losses Rogers 4350B substrate. The proposed circuit can wake-up starting from a fully discharged state and operate at distances up to $9.70 \mathrm{~m}$ from a $2 \mathrm{~W}$ ERP source in the UHF 865$868 \mathrm{MHz}$ frequency band, with a power sensitivity of $-16 \mathrm{dBm}$, while performing temperature sensing and radio-frequency communication. If an on-board battery is added, the batteryassisted solution is able to work as far as $15 \mathrm{~m}$ from the interrogator reaching the highest sensitivity of $-\mathbf{1 7 . 8} \mathrm{dBm}$ while the energy harvesting circuits replenishing the battery.
\end{abstract}

Keywords-RF energy harvesting, wake-up radio, RFID, micropower circuit, environmental monitoring.

\section{INTRODUCTION}

The radio-frequency identification systems (RFID), initially developed only for identification, are becoming more appealing over the years with the addition of sensing and positioning capabilities [1], enabling businesses and consumers to identify, locate, authenticate and engage each item in a new world called RAIN-RFID [2].

These systems generally consist of small, battery-free and flexible devices called tags which are energized and interrogated by readers. Once woken up, each node responds back to interrogators an identification number whereby the backscattering modulation technique [3] by following the Electronic Product Code (EPC) Class 1 Gen 2 standard [4]. Among RFID systems, higher communication range is achieved by Ultra High Frequency (UHF) RFID, in passive, active and semi-active configurations [5].

Active tags require continuous power to operate, provided by an on-board battery. Although, this solution allows a higher read-range in the order of tens of meters at the expense of a constant batteries maintenance for recharge or replacement. On the other hand, passive tags harvest the power from the radiofrequency source and collect all energy in a storage capacitor, properly dimensioned to accomplish the RFID $\mathrm{read} /$ write operations and sensing. Since the RF available power as a function of the distance can be as low as tens of $\mu \mathrm{W}$, this system typically has a forward limited communication range up to few meters [6]. In general, passive tags do not embed sensing capabilities.

The improvement on read-range distances of passive RFID

This work was supported in part by the ECSEL JU through the European Union's H2020 Research and Innovation Program and Germany, Slovakia, Netherlands, Spain, and Italy, under grant agreement no. 737434. tags was made possible by the reduction of energy consumption of circuits, in addition to the higher sensitivity of both readers and tags. J. R. Smith et al, in 2005, were the pioneers in passive RFID tag extended with sensing capabilities. They reported the first use of ID modulation to embed environmental information within the EPC standard [7]. The Wireless Identification and Sensing Platform (WISP) was improved by P. Sample et al. in 2007 [8] and 2008 [9], by developing the first low-cost battery-free programmable UHF-RFID node with sensors, where the EPC Class 1 Gen 2 protocol is firmware implemented in a microcontroller. WISP is able to work up to 4.5 meters from the RF source using only a single dipole antenna.

D. De Donno et al, in 2014, presented a RFID augmented module for smart environmental sensing (RAMSES) [10] using the findings of the WISP project. The node implements two separated UHF antennas, for RF energy harvesting purposes and wireless sensor data communication, through RFID standard. The system is able to operate up to 10 meters in a battery-less solution while the read range is extended to 22 meters in a battery-assisted configuration. On the other hand, as limitation, the usage of two different antennas makes the prototype expensive in terms of occupied area and the RFID protocol implemented by Monza X-2K, has lower flexibility compared to WISP firmware one.

Furthermore, in addition to research activity, many industries are working on integrated UHF-RFID sensory tag solutions. In particular, the most important are SL900A chip tag by Austria Micro System (AMS) [11], Easy2Log tag by CAEN RFID [12] and EVAL01-Fenix-RM by Farsens [13].

Many flexible solutions are available on the market such as SPS1M002 by On Semiconductor [14], the sensor node DogBone by Smartrac [15] and others [16].

Hence, this work aims to update the last version of WISP [9] with improved hardware architecture, based on [17] and on a novel dual-output rectenna, able to operate at long read-range distances compliant with the regulation provided by the

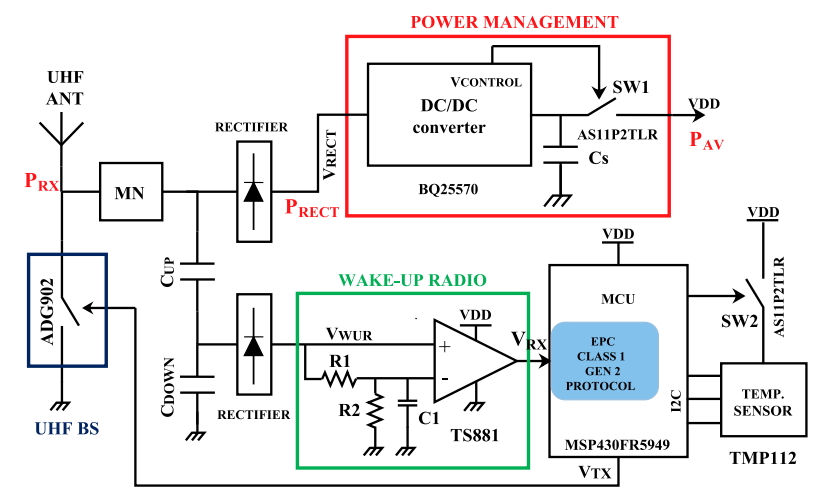

Fig. 1. UHF-RFID passive sensor node architecture. 


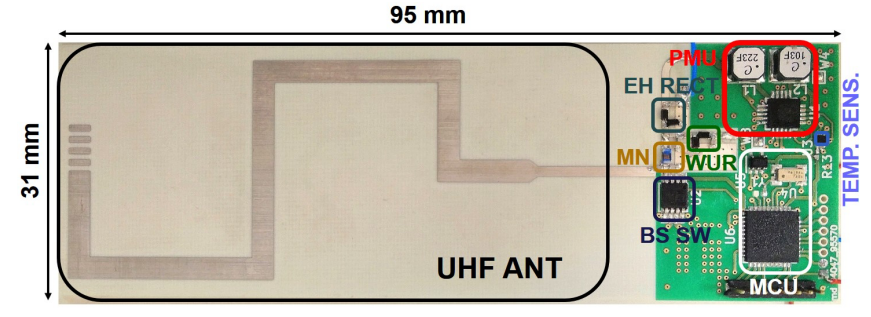

Fig. 2. UHF-RFID passive sensor node PCB board.

European Telecommunication Standard Institute (ETSI) and full working within $865-868 \mathrm{MHz}$ frequency band [18].

The obtained performances are comparable with integrated solutions available in the market and in academy albeit the EPC Class 1 Gen 2 protocol is still firmware implemented in a microcontroller. The system is easily programmable and maintains high flexibility allowing operation at a low level within the execution of the protocol phases.

The paper is organized as follow: Section II describes the architecture and design trade-off used to develop the sensor platform blocks. Subsequently in Section III, the measurements results are presented in order to evaluate the overall system performance, followed by Conclusions in Section IV.

\section{ARCHITECTURE AND DESIGN TRADE-OFF}

The hardware architecture of the proposed passive sensor tag is depicted in Fig.1. The node is based on a single UHF monopole used for both RF energy harvesting and Wake-up radio capabilities. The RF path is split in two different parts through a capacitive divider. The rectified voltage on the main power conversion output port is boosted by the onboard DC/DC converter which performs a maximum power point tracking (MPPT) of the RF source and supplies the load circuitry when sufficient energy is available on the storage capacitor $\mathrm{C}_{\mathrm{s}}$.

A voltage monitor embedded in the $\mathrm{DC} / \mathrm{DC}$ converter allows to perform the load power gating (SW1) between high and low threshold voltages according to load requirements.

On the RF side, a very limited part of the incoming power is rectified to provide wake-up signals to a microcontroller unit (MCU) placed for the firmware implementation of the EPC Class 1 Gen 2 protocol, which is based on the open-source version provided by the WISP project [19]. With respect to a standard tag, the MCU attaches the temperature data information to the EPC frame during the standard communication exchange.

Since the power consumption of the overall system plays an important role for the performance evaluation, an additional switch (SW2) is used to turn off the external sensor during inactivity periods, in order to reduce unnecessary quiescent current consumption. The prototype has been implemented in a 4-layer low-losses Rogers 4350B printed circuit board (PCB) shown in Fig.2. This architecture is an enhancement of a previous system described in [20] that, differently from this version, does not manage sensors, has no backscattering capabilities for RFID communication and includes only hardware for UWB localization.

\section{A. RF Front End}

A single rectenna (rectifying antenna), composed of a UHF meandered monopole (1.8 dBi of gain), is designed and tuned

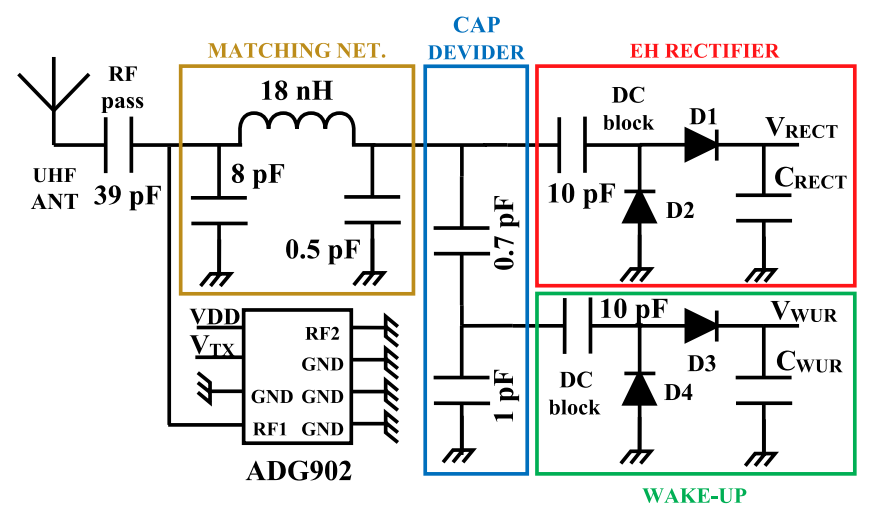

Fig. 3. RF Front End.

in the European RFID band $865-868 \mathrm{MHz}$ combined with a matching network and rectifiers, Fig 3 . The rectenna features also a wake-up radio (WUR) implementation, without recurring to a dedicated second antenna and therefore reducing the overall tag dimensions. The purpose of the WUR is to bound the employment of the power-consuming components of the tag to the sole active phase.

The MCU can therefore be kept in an idle, listening state and be waken-up by the low-latency WUR when the specific tag is interrogated. The input for the WUR implementation has therefore to be derived from the UHF received signal. To this extent, the most obvious solution would be that of providing an input for the WUR by splitting the dc voltage at the output of the rectifier with a conventional resistive divider. However, this solution is by definition lossy, as it involves lossy components. Given the extremely low values of power available at the high distances foreseen for the system, losing even only few $\mu W$ of power is not acceptable. For this reason, we resort to the solution presented in [21] where the voltage is split at RF level, by means of a capacitive divider. In this way, only reactive components are involved, resulting in almost no losses, except for the parasitic ones due to the nonideal capacitors. Through the capacitor divider, only a small portion of the received power is delivered to the WUR side, where tens of $\mathrm{mV}$ are enough to decode the data received. This choice, makes the entire network unbalanced to the power conversion side for more efficient energy harvesting. The rectifiers stages are based on Skyworks SMS7630079LF Schottky diodes, with threshold voltages lower than $240 \mathrm{mV}$, specifically designed for these purposes.

Finally, the backscattering signal sent back to the reader through the MCU is modulated by the ADG902 switch (Analog Devices) which has good RF performance up to $1 \mathrm{GHz}$ and low quiescent current consumption to operate.

In order to perform backscattering, the switch changes the antenna impedance by switching between ground short circuit and matched states offered by the matching network.

\section{B. Digital base-band Tag operation}

Once the node is energized and circuits are powered, it goes in an idle state waiting for the wake-up signal coming up by the reader. The received modulated forward link signal is cleaned with a data slicer, Fig. 1, based on an ultra-low power comparator (TS881 by STMicroelectronics) which brings the input weak signal to correct digital voltages used by the microcontroller. It worth to mention that R1, R2 and $\mathrm{C} 1$ have 


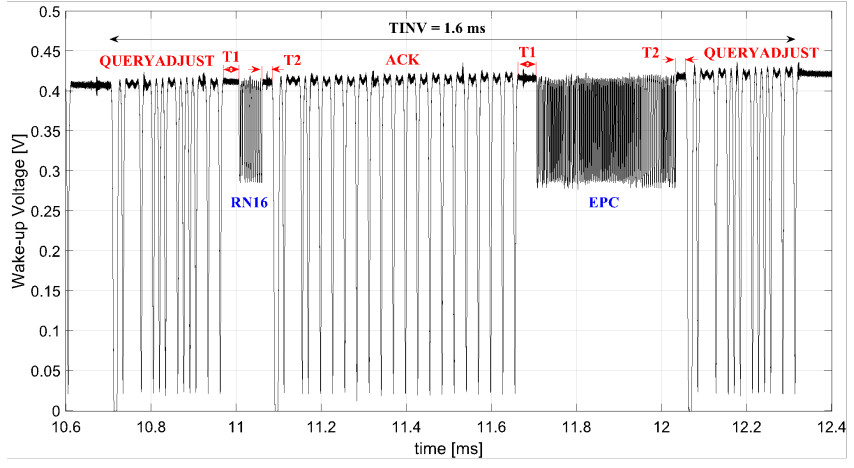

Fig. 4. Tag Inventory-round with $\mathrm{Q}=0$, and Tag population set to 1 , since there is only one node to identify.

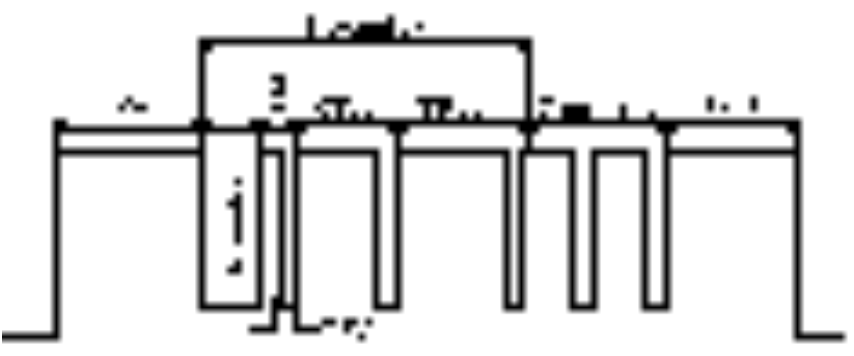

Fig. 5. Reader to Tag communication parameters.

to be properly chosen in order to filter out the unwanted wake-up requests. In particular, the MPPT practiced by the $\mathrm{DC} / \mathrm{DC}$ converter influences the correct detection of the wake-up signal and commutates the comparator output. The filter is placed with a resistive divider with $R 1=R 2=$ $1 M \Omega$, so that the comparator negative input is the half of the positive one in order to deal fast signal changes. The capacitance value of $C 1=10 \mathrm{nF}$ is chosen accordingly to the forward link communication data-rate of $128 \mathrm{Kbps}$. Hence, the data frame is ready to be processed by the microcontroller which is in charge of implementing the EPC Class 1 Gen 2 standard protocol with a firmware derived from the open source code provided by the WISP project [19] and adapted to work with European standards. The interrogator manages the Tag population using the Select command to define a specific group of nodes, then it starts an inventory round (Fig. 4) with a Query command which contains the slot counter number $\mathrm{Q}$ (defined between 0 to 15 ).

This number is used to run a counter with a random value between 0 to $2^{Q}-1$. Through QueryAdjust commands, the Q number can be brought to zero to speed-up the inventoryround enabling the node to send back the 16-bit random number RN16 (Fig.4). The RN16 allows to have a random access to the channel and it is followed by an acknowledge command where the same value is placed. Finally, to end the inventory round, the tag replies with the Electronic Product Code (EPC) used as Tag identification.

During the forward link communication, the MCU sets its internal fast clock of $16 \mathrm{MHz}$ to detect the pin changes on the receive channel in a real-time configuration and classifies the commands in order to define the next operation. If a reply is expected, the reverse link communication is managed with a $8 \mathrm{MHz}$ clock frequency, for lower power consumption, since it is not time critical as the previous phase. The WISP project, initially, was intended for United States readers where the constrains in terms of transmitted power and link frequencies
TABLE I. MAIN UHF-RFID CONFIGURATION PARAMETERS

\begin{tabular}{|c|c|c|}
\hline Name & Value & Explanation \\
\hline $\mathrm{T}_{\mathrm{CW1}}$ & $3.8 \mathrm{~ms}$ & Continuous wave \\
\hline Delimiter & $12.5 \mu s$ & Start of frame \\
\hline Tari & $16.67 \mu s$ & Modulation depth \\
\hline$\overline{P W}$ & $8.335 \mu s$ & "0" duration \\
\hline $\mathbf{R T}_{\mathrm{CAL}}$ & $41.7 \mu s-50 \mu s$ & Pulsewidth \\
\hline TR $_{\text {CAL }}$ & $45.8 \mu s-150 \mu s$ & "0" + " 1 " duration \\
\hline $\mathbf{T}_{\mathrm{CW} 2}$ & undefined & Tag backscatter phase \\
\hline BLF & $436 \mathrm{Kbps}$ & Backscat. link freq. \\
\hline FLF & $128 \mathrm{Kbps}$ & Forward link freq. \\
\hline DR & $64 / 3$ & Divide Ratio \\
\hline Rev. Mod. & FM0 & Reverse signal coding \\
\hline $\begin{array}{c}\text { T1 } \\
\mathrm{RT}_{\mathrm{CAL}}: 41.6 \mu \mathrm{s} \\
\mathrm{RT}_{\mathrm{CAL}}: 50 \mu \mathrm{s}\end{array}$ & $\begin{array}{c}30.5 \mu s-52.8 \mu s \\
37 \mu s-63 \mu s\end{array}$ & $\begin{array}{c}\text { Time from } \\
\text { Interrogator } \\
\text { transmission to Tag }\end{array}$ \\
\hline T2 & $7.5 \mu s-50 \mu s$ & $\begin{array}{c}\text { Time from Tag reply } \\
\text { to Interrogator } \\
\text { transmission }\end{array}$ \\
\hline
\end{tabular}

are different from European versions (4W EIRP in 902-928 $\mathrm{MHz}$ frequency band). For this reason, significant changes have been done to adjust the firmware for operation with European readers. Table I and Fig.5, summarizes all set of parameters used to interact with a European reader using the open-source WISP firmware. In particular, the timing T1 and T2, shown in Fig. 4, are protocol defined and have been properly set on the Tag side to make it operate properly.

In addition, the EPC, by design choice, contains two bytes with the temperature sensor data information. When the tag is powered-up the sensor requires $35 \mathrm{~ms}$, in the worst condition, to have an updated version of the temperature data, subsequently stored in the non-volatile memory of the MCU. Whereas, an inventory round ends in less than $1.6 \mathrm{~ms}$ and the sensor conversion time defines the minimum Tag Activation time $\left(T_{A C T}\right)$.

\section{Power management and Energy budget}

The tag operation is the direct consequence of the power management choices [22]. In Fig. 6, the load is activated when the storage capacitor Cs reaches the high threshold voltage $V H=2.2 \mathrm{~V}$ and subsequently switched off once it falls down to the low threshold voltage $V L=1.85 \mathrm{~V}$ (chosen by considering the MCU minimum voltage supply of $1.80 \mathrm{~V}$ ). The load power gating is performed using the switch SW1 controlled by the voltage monitor implemented into the power management unit. The stored energy from a fully discharged state is $E_{O N}=1 / 2 C s V H^{2}$, collected in $T_{O N}=$ $E_{O N} / P_{A V}$, where $P_{A V}$ is the available power as output of the DC/DC converter:

$$
P_{A V}=D_{C W} \eta \alpha P_{R X}
$$

Starting from the UHF antenna received power $\left(P_{R X}\right), \eta$ and $\alpha$ are the efficiencies of the rectifier stage and power management unit respectively. In a real scenario, both efficiencies depend on the received power with a non-linear behaviour. To simplify the model, the values taken into 


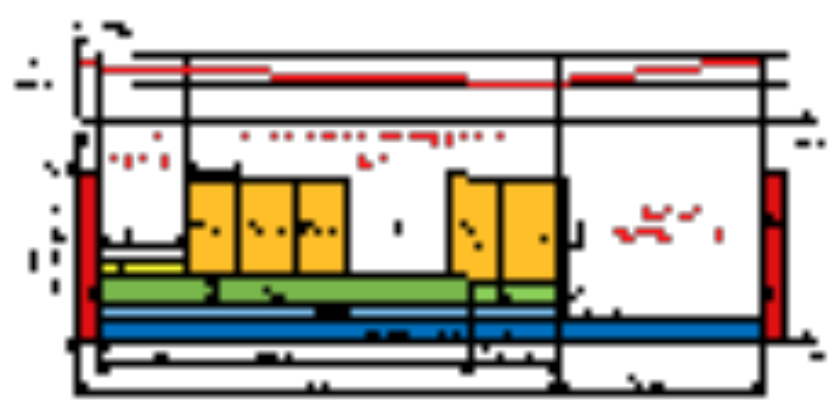

Fig. 6.Tag energy consumption profile. It has been supposed that the reader performs a Single Target Inventory in Session 0.

TABLE II. MAIN UHF-RFID ENERGY-BUDGET PARAMETERS

\begin{tabular}{|c|c|c|}
\hline Param. & Value & Explanation \\
\hline $\boldsymbol{T}_{\boldsymbol{S T A R T}}$ & $500 \mu \mathrm{s}$ & Load Power-up time \\
\hline $\boldsymbol{T}_{\boldsymbol{A C T}}$ & $35.57 \mathrm{~ms}$ & Tag Activation Time \\
\hline $\boldsymbol{T}_{\boldsymbol{I N V}}$ & $1.6 \mathrm{~ms}$ & Inventory Round Time \\
\hline $\boldsymbol{I}_{\boldsymbol{I N V}}$ & $1.2 \mathrm{~mA}$ & MCU average current consumpt. \\
\hline $\boldsymbol{T}_{\boldsymbol{I D L E}}$ & $10 \mathrm{~ms}$ & Max Tag idle time \\
\hline $\boldsymbol{I}_{\boldsymbol{C O M P}}$ & $210 \mathrm{nA}$ & TS881 current consumption \\
\hline $\boldsymbol{I}_{\boldsymbol{S L E E P}}$ & $300 \mathrm{nA}$ & MCU current consumpt. in LPM4 \\
\hline $\boldsymbol{P}_{\boldsymbol{D C D C}}$ & $1.38 \mu \mathrm{W}$ & DC/DC power consumption \\
\hline $\boldsymbol{P}_{\boldsymbol{S W 1}}$ & $200 \mathrm{nW}$ & SW1 power consumption \\
\hline $\boldsymbol{T}_{\boldsymbol{C O N V}}$ & $35 \mathrm{~ms}$ & TMP112 conversion time \\
\hline $\boldsymbol{I}_{\boldsymbol{C O N V}}$ & $40 \mu \mathrm{A}$ & TMP112 temp. conversion current \\
\hline $\boldsymbol{I}_{\boldsymbol{I I C}}$ & $15 \mu \mathrm{A}$ & TMP112 I2C current consumpt. \\
\hline $\boldsymbol{T}_{\boldsymbol{B I T}}$ & $2.5 \mu \mathrm{s}$ & I2C bit time (@ 400 Kbps) \\
\hline $\boldsymbol{N}_{\boldsymbol{B I T}}$ & 28 & 1 byte address +2 bytes data \\
\hline
\end{tabular}

account are referred, as worst case, to the lower $P_{R X}$ necessary to ensure the Tag start-up ( $\eta=28 \%, \alpha=65 \%$ ).

Moreover, the value of continuous wave duty-cycle $D_{C W}=$ $66 \%$ has been used to consider the periodic standard-defined power holes performed by the interrogator used in the tests.

Hence, the storage capacitor Cs has to be defined according to the Tag active time $\left(T_{A C T}\right)$ required in order to accomplish the inventory rounds and store the temperature sensor information. For this reason, from the energy budget point of view, the $T_{A C T}$ must be:

$$
T_{A C T} \geq T_{S T A R T}+T_{C O N V}+N_{B I T} T_{B I T}=35.57 \mathrm{~ms}
$$

in order to store a new temperature value.

All parameters in terms of devices current consumption and timings are illustrated in Table II. When the $T_{A C T}$ is ended, the tag load is switched off while only the power management unit is still working to harvest the energy. Therefore, the minimum available power required to charge the storage capacitor is $P_{A V \min }>P_{D C D C}+P_{S W}=1.58 \mu W$ which represents the quiescent power consumption. To simplify the following considerations, it has been assumed the pejorative approximation for the storage capacitor voltage $\left(V_{S}=V_{H}\right)$. The total amount of energy used for tag operation in $T_{A C T}$ is defined in three different states as:

$$
\Delta E=E_{S T A R T}+E_{I D L E}+E_{I N V 1, N}
$$

where:

- $E_{S T A R T}$ : load power-up state energy;

- $E_{I D L E}:$ idle state energy;

- $E_{I N V 1, N}$ : inventory-rounds state energy;

- $\quad N=\left(T_{A C T}-T_{I D L E}-T_{S T A R T}\right) / T_{I N V}$ number of inventory rounds per $T_{A C T}$.

Once the MCU is activated $\left(E_{S T A R T}=391 \mathrm{~nJ}\right)$, it goes into low power mode 4 (LPM4) and the Tag energy consumed $\left(E_{I D L E}\right)$ is calculated as follow:

$$
E_{I D L E}=V_{H} T_{I D L E}\left[I_{S L E E P}+I_{C O N V}+I_{C O M P}\right]=891 n J
$$

$T_{I D L E}$ is not well predictable, because it depends on where the load power-up instant falls during the reader interrogation. The time between two inventory-rounds cycle (10ms), defined by the protocol, determines the worst case for $T_{I D L E}$. Subsequently, the reader starts the inventory rounds and the node goes into the most intensive-energy state $\left(E_{I N V 1, N}\right)$, where it consumes:

$$
\begin{aligned}
E_{I N V 1, N} & =V_{H}\left[I_{C O M P} T_{A C T}+I_{C O N V}\left(T_{C O N V}-T_{I D L E}\right)+\right. \\
& \left.+I_{I 2 C} N_{B I T} T_{B I T}+N I_{I N V} T_{I N V}\right]=68.4 \mu J
\end{aligned}
$$

The energy consumption of SW2 during $T_{I N V}$ has been neglected since it is hundreds times lower compared to other contributions.

Hence, the overall Energy consumed by the tag in one activation period is $\Delta E=69.7 \mu \mathrm{J}$. The impact of the idle state and start-up are negligible if compared to the inventory rounds state where the tag is read $\mathrm{N}$ times consecutively.

The energy information has been collected in order to define the appropriate storage capacitor for the system:

$$
C_{S}=\frac{2 \Delta E}{V_{H}^{2}-V_{L}^{2}}=98.3 \mu F
$$

A capacitor of $100 \mu F$ has been chosen according to the consideration over reported. Now, it is possible to calculate the tag charge time as a function of the available power:

$$
T_{C H A R G E}=\Delta E /\left(P_{A V}-P_{D C D C}-P_{S W}\right)
$$

which is used to evaluate the performance of the energy harvesting blocks by introducing the Duty Cycle parameter:

$$
D_{A C T}=T_{A C T} /\left(T_{A C T}-T_{C H A R G E}\right)
$$

Increasing the storage capacitor $C_{S}$, enlarge the active time period and consequently the inventory-rounds per cycle $(\mathrm{N})$. However, the charge time rises in the same way without affecting the Duty Cycle and the number of tags read per time unit. Therefore, the idea is to set the minimum capacitance value to accomplish the required tasks during the active period in order to have readings well distributed in time.

The number of reads per second can be computed as follow:

$$
\text { Number of Reads } / \mathrm{sec}=N /\left(T_{A C T}+T_{C H A R G E}\right)
$$

All parameters used to evaluate the RF energy-harvesting performance of the tag [23], in terms of duty-cycle operation and read-range are discussed in the next section with the comparison between theoretical and experimental results. 


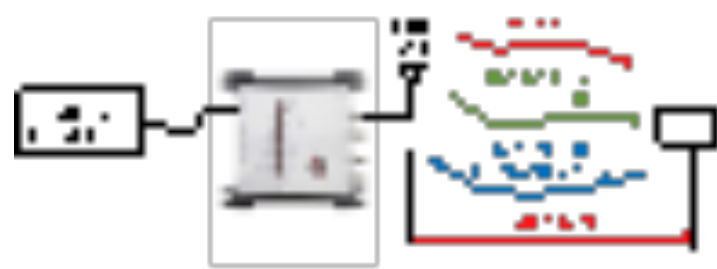

Fig. 7. Test bench for the UHF-RFID tag performance measurements.

\section{EXPERIMENTAL RESULTS}

The test bench for measurements used in indoor environment is shown in Fig. 7. It is based on the reader Speedway R420 by Impinj connected to the antenna Far Field ( $8.5 \mathrm{dBi}$ of gain) of the same company jointly with the Impinj Item Test software used for Tag Inventory and Access. To be ETSI compliant, the maximum transmitted power of $2 \mathrm{~W}$ ERP has been used. The reader is configured to perform Inventory rounds with Single Tag Inventory mode in Session 0, which represents the maximum reading throughput.

\section{A. RF Energy Harvesting performance}

First of all, to characterize the Tag performance, the Antenna behavior combined with the matching network and rectifiers was evaluated. Simulation and measurements of the rectified voltages as a function of the received power $\left(P_{R X}\right)$ are shown in Fig. 8. The unbalanced capacitor divider used to split the Wake-up radio $\left(V_{W U R}\right)$ and energy harvesting $\left(V_{R E C T}\right)$ blocks brings to have higher voltages where the energy has to be collected and managed to supply the tag. Hence, the wake-up radio has a reduced voltage range where tens of $\mathrm{mV}$ are enough to correctly detect the commands sent by the reader. The tag sensitivity and consequently the maximum readrange reachable are presented in Tab. III. As measurement approach [23], in order to evaluate the first activation range, the platform has been placed at $10 \mathrm{~m}$ of distance from the reader and moved closer it until the node has been poweredup $\left(T_{O N}=23 \mathrm{~s}\right.$ at $\left.7 \mathrm{~m}\right)$. Then, starting from the same position, the distance has been increased with incremental

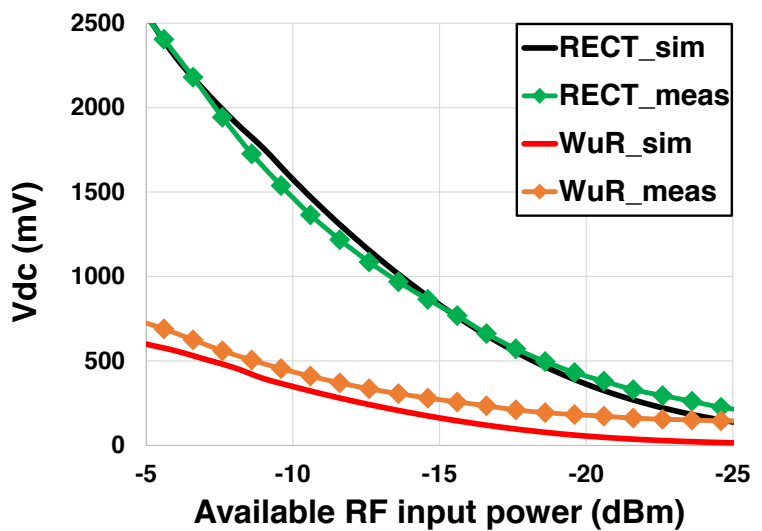

Fig. 8. Comparison between measured and modelled open circuit voltages at the output of the rectifier and wake-up radio sections.

TABLE III. MAIN UHF-RFID CONFIGURATION PARAMETERS

\begin{tabular}{|c|c|c|}
\hline Tag Parameter & Battery-less & Battery-assisted \\
\hline Sensitivity $[\mathbf{d B m}]$ & -16 & -17.8 \\
\hline Max read-range $[\mathrm{m}]$ & 9.70 & 15 \\
\hline First activation $[\mathrm{m}]$ & 7 & $/$ \\
\hline
\end{tabular}

step of $1 \mathrm{~m}$ up to the maximum distance $(9.70 \mathrm{~m})$ where the tag stopped operating due to insufficient incoming power. The sensitivity is defined as the minimum amount of received power required to keep alive the power management unit and performs the EPC Gen 2 Class 1 protocol answering back to the interrogator. In the battery-less configuration, the tag has a sensitivity of $-16 \mathrm{dBm}$ with a read-range of $9.70 \mathrm{~m}$, which is limited by the forward power transfer. If an on-board battery is present (battery-assisted configuration) the sensitivity, considered only for data link communication, falls to $-17.8 \mathrm{dBm}$ with a maximum read-range of $15 \mathrm{~m}$.

In this case, the limitation is given by the reverse link where few $\mathrm{mV}$ of Tag received voltage on the wake-up radio, are not sufficient to demodulate the data stream. Moreover, on the reader side, the power incoming from the backscattered signal is weak and close to the interrogator sensitivity of -70 $\mathrm{dBm}$ which represents another limit on the read-range.

\section{B. Tag duty-cylce and charge time}

A set of inventory-rounds have been run, for all tag positions starting from $1 \mathrm{~m}$ up to $9.70 \mathrm{~m}$, to collect the timestamps of all acquisitions in order to evaluate the charge time and subsequently the duty-cycle. All measurements are reported in Fig. 9 and compared with analytical results of Eq. (7) and Eq. (8). The measured results are consistent compared with the analytical ones, except slight differences due to the multipath effects on the transmitted signal which is reflected by the obstacles. On the tag side, all contributions can be added in phase, by increasing the received power (this is the case between $7 \mathrm{~m}$ to $9.70 \mathrm{~m}$ ), or out of phase by lowering the received power with the consequence of slower charge time (starting from $1 \mathrm{~m}$ up to $7 \mathrm{~m}$ ).

\section{Read-Rate and Environmental sensing}

In all RFID applications, during the requirements definition, one of the most popular parameters is the read-rate, which is the number of tags read per time unit. The presented batteryless sensor Tag has a read-rate of 2908 reads per minute at $1 \mathrm{~m}$ decreasing down to 48 reads per minute at $9.70 \mathrm{~m}$, Fig. 10. The read-rate can rise up to 725 tags per second if the node is equipped with an on-board battery without limitation given by the wireless power transfer. As discussed in the previous section, the EPC contains the sampled temperature data which is updated every $T_{A C T}$ period where one temperature conversion is performed and stored. So, all

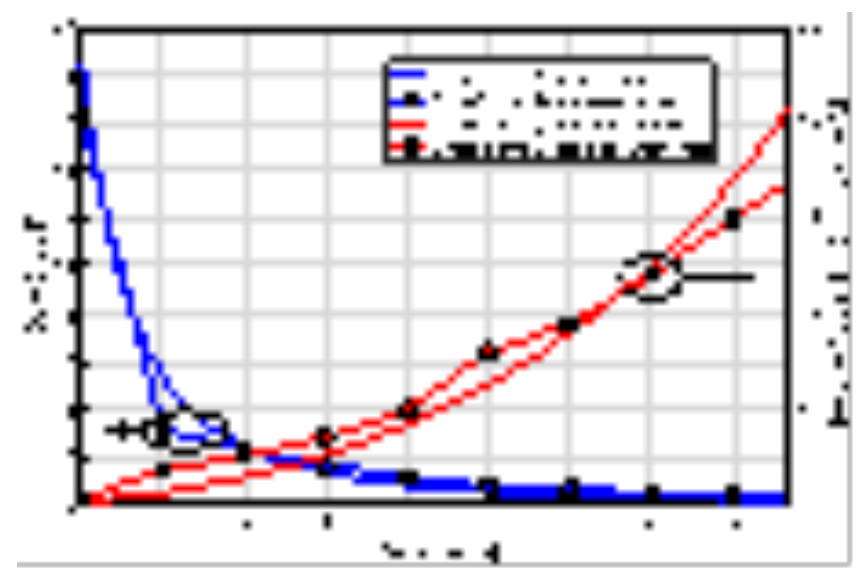

Fig. 9. Comparison between modelled and measured Tag duty-cycle and charge time. 


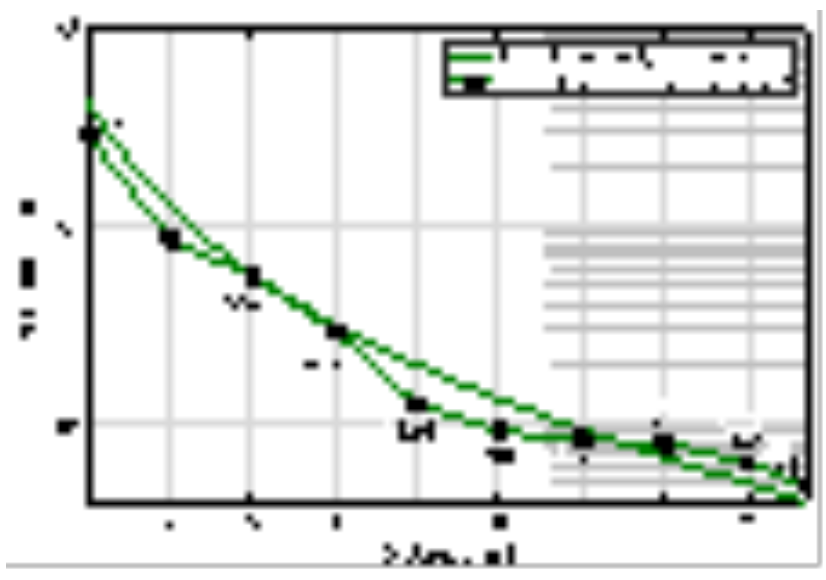

Fig. 10. Comparison between measured and modelled Battery-less tag read rate as a function of the distance from the reader antenna.

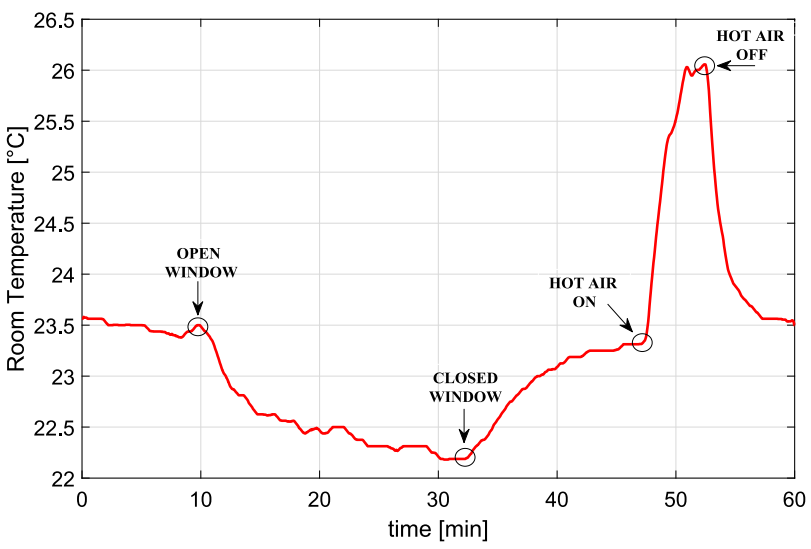

Fig. 11. Room temperature recording.

inventory-rounds $(\mathrm{N})$ executed during $T_{A C T}$ contains the previous temperature recorded in $T_{A C T-1}$ in order to preserve the energy consumption for faster re-activation.

In Fig. 11, $1 \mathrm{~h}$ of recorded temperature is shown where several external disturbances have been applied to test the sensor. After 10 minutes a window has been open and subsequently closed at minute 30 with a temperature variation of about $1.5^{\circ} \mathrm{C}$. Then, a hot air machine has brought the room temperature to $26^{\circ} \mathrm{C}$ in few minutes.

\section{CONCLUSION}

A new design of battery-less UHF-RFID sensor tag has been presented for all applications which requiring high read-rage solutions with sensing capabilities.

The read-rate performance makes the platform suitable for localization purposes even when real-time tracking of goods and people is requested. The node is European reader friendly and has the advantages of the WISP tag [9] where the RFID protocol is firmware implemented, thus allowing operational flexibility with an improved low power circuit design used to extend the working range. Moreover, in order to overcome the bottleneck of the forward link communication, ASIC solutions such as [24][25] can be used to replace the power management unit for even better read-range.

\section{REFERENCES}

[1] P. Adamcova, Z. Tobes, "UHF RFID Technology and its Application" $17^{\text {th }}$ International Conference Radioelektronika, June 2007.
[2] RAIN-RFID, https://rainrfid.org/.

[3] Pavel V. Nikitin, K. V. S. Rao, "Theory and Measurement of Backscattering from RFID Tags", IEEE Antennas and Propagation Magazine (Volume 48, Issue: 6, Dec. 2006).

[4] EPCGlobal, "EPC Radio-Frequency Identity Protocols Gneneration-2 UHF RFID", Version 2.0, Nov. 2013.

[5] M. Ayoub Khan, M. Sharma, Brahmanandha Prabhu R, "A Survay of RFID Tags", International Journal of Recent Trends in Engineering, Vol. 1, No. 4, May 2009.

[6] Pavel V. Nikitin, K. V. S. Rao, "Performance Limitations of Passive UHF RFID Systems", IEEE Antennas and Propagation Society International Symposium, Oct. 2006.

[7] J. R. Smith, B. Jiang, S. Roy, M. Philipose, K. Sundara-Rajan and A. Mamishev, "ID Modulation: Embedding Sensor Data in a RFID Timeseries", International Workshop on Information Hiding, Vol. 3727, Nov. 2005.

[8] A. P. Sample, D. J. Yeager, P. S. Powledge, J. R. Smith, "Design of Passively-Powered, Programmable Sensing Platform for UHF RFID Systems", IEEE International Conference on RFID, Grapevine (TX, USA), March 2007.

[9] A. P. Sample, D. J. Yeager, P. S. Powledge, A. V. Mamishev, J. R. Smith, "Design of an RFID-Based Battery-Free Programmable Sensing Platform", IEEE Transaction on Instrumentation and Measurement, Vol. 57, No. 11, Nov. 2008.

[10] D. D. Donno, L. Catarinucci and L. Tarricone, "RAMSES: RFID Augmented Module form Smart Environmental Sensing", IEEE Transaction on Instrumentation and Measurement, Vol. 63, No. 7, July 2014.

[11] UHF RFID Gen 2 SL900A Sensory Tag IC [Online], 2013, Available: https://ams.com/.

[12] EasyToLog RFID Tag [Online], 2013, Available: https://www.caenrfid.com/en/.

[13] EVAL01-Fenix-RM temperatur sensor board [Online], Available: https://www.farsens.com

[14] SPS1M002, Smart Passive Sensor for Direct Moisture Sensing [Online], 2017, Available: https://www.onsemi.com.

[15] Smartrac DogBone sensor node [Online], Available: https://www.smartrac-group.com/

[16] G. Zhang, D. Wu, J. Jia, W. Gao, Q. Cai, W. Xiao, L. Yu, S. Tao and Qi Chu, "Architecture Chacteristics and Technical Trends of UHF RFID Temperature Sensor Chip", Active and Passive Electronic Components, Article ID 9343241, 2018.

[17] D. Fabbri, M. Pizzotti, and A. Romani, "Micropower Design of an Energy Autonomous RF Tag for UWB Localization Applications", IEEE International Symposium on Circuits and Systems (ISCAS), May 2018, pp. 1-5.

[18] ETSI EN 302 208, V3.1.0 (2016-02) [online], Available: https://www.etsi.org/.

[19] WISP Project repository [Online], Available: http://www.wispsensor.net.

[20] D. Dardari, N. Decarli, D. Fabbri, A. Guerra, M. Fantuzzi, D. Masotti, A. Costanzo, A. Romani, M. Drouguet, T. Feuillen, C. Raucy, L. Vandendorpe and C. Craeye, "An Ultra-wideband Battery-less Positioning System for Space Applications", submitted to IEEE RFIDTA-2019.

[21] M. Fantuzzi, M. Del Prete, D. Masotti and A. Costanzo, "Quasiisotropic RF energy harvester for autonomous long distance IoT operations," 2017 IEEE MTT-S International Microwave Symposium (IMS), 2017, pp. 1345-1348.

[22] A. Costanzo, D. Dardari, J. Aleksandravicius, N. Decarli, M. Del Prete, D. Fabbri, M. Fantuzzi, A. Guerra, D. Masotti, M. Pizzotti and A. Romani, "Energy Autonomous UWB Localization", IEEE Journal of Radio Frequency Identification, Vol. 1, No. 3, Sept. 2017.

[23] Pavel V. Nikitin and V. S. Rao, "Antennas and Propagation in UHF RFID Systems", IEEE International Conference on RFID, Las Vegas, NV, USA, May 2008.

[24] M. Dini, A. Romani, M. Filippi and M. Tartagni, "A Nanocurrent Power Management IC for Low-Voltage Energy-Harvesting Sources", IEEE Transaction on Power Electronics, Vol. 31, Issue 6, June 2016.

[25] M. Pizzotti, L. Perilli, M. Del Prete, D. Fabbri, R. Canegallo, M. Dini, D. Masotti, A. Costanzo, E. Franchi Scarselli and A. Romani, "A LongDistance RF-Powered Sensor Node with Adaptive Power Management for IoT Applications, Sensors 2017, 17(8), 1732 